\title{
Integrated cutting and production planning: a case study in a home textile manufacturing company
}

\author{
Elsa Silva, Cátia Fernandes, José F. Oliveira, Maria Antónia Carravilla
}

INESC TEC, Faculdade de Engenharia, Universidade do Porto

\begin{abstract}
In this paper we consider the problem of minimizing the waste of textile material in a Portuguese home textile manufacturing company. The company has a vertical structure covering the different production stages of the home textile, from weaving until the finished products. Production planning comprises different decisions: the definition of the widths and lengths of the fabric rolls to be produced, the number of fabric rolls to be used from stock or purchased and the definition of the cutting patterns to be applied to each width of the fabric roll, so that the waste is minimized. We propose a MIP model, solved by a column generation method, to tackle the problem.
\end{abstract}

\section{Introduction}

The study of the production planning problem integrated with the cutting process on a home textile manufacturing company is the focus of this paper. The company is located in the north of Portugal and the final products are sheets, pillowcases and duvet covers.

The production process of this textile company has the following phases: weaving, dyeing and/or printing, cutting, sewing and packaging. The last two phases are not considered in this study since they do not contribute to the waste of textile material. Between each pair of the remaining phases there is an intermediate stock to be used in a subsequent production phase, arising either from a finished product of an upstream phase or purchased from an external supplier. The production process is illustrated in Figure 1.

In the weaving process, the fabric rolls are produced in industrial looms. The rolls are then dyed in a bathtub where they acquire the final color. In the printing phase drawings of one or more colors are reproduced on the fabric rolls through a serigraphy process. This process is optional as the customer may decide on plain or patterned products.

The process ends at the cutting phase where the fabric rolls are unrolled and folded in layers on a cutting table and are cut following a cutting pattern (see Figure 2 (a)). A cutting pattern represents the layout of the pieces on the layer. 
Fig 1. Production process (Weaving-Cutting).

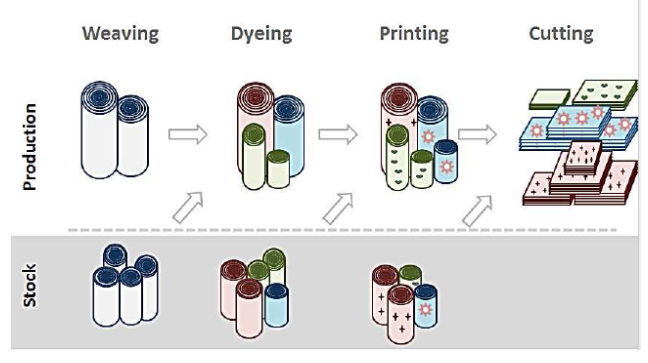

Fig 2. Fabric roll on the cutting table.

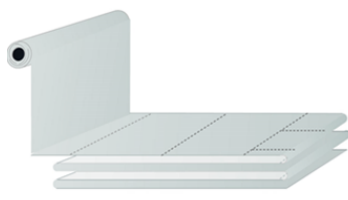

(a)

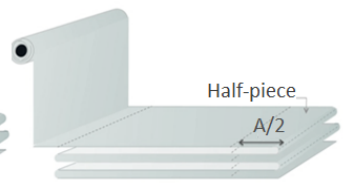

(b)

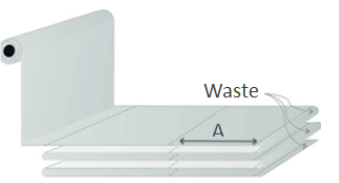

\section{Cutting Process}

The layer is cut manually with a saw, thus the cutting patterns must be of type guillotine. In a guillotine type cutting pattern the cuts are made along the entire length/width of the cutting pattern and cannot stop in the middle of the path (see Figure 3). Another important characteristic of the cutting patterns is the number of stages, i.e. the number of cuts with successively different directions that must be performed by the saw, in order to cut the pieces as planned in the cutting pattern. The cutting patterns considered by the company are at most three-stage, eventually with an additional cut performed to separate the pieces from waste, called trimming. An example of a three-stage cutting pattern with trimming is presented in Figure 3.

The technique used to spread the fabric rolls on the cutting table enables to cut "half-pieces" by using the folds between layers (see Figure 2 (b)). This is however not possible for orders in which a high accuracy in the dimensions of the pieces is needed. In this case a cut at the fabric roll ends is required, in order to divide the layers, producing additional waste (see Figure 2 (c)).

Fig 3. Three-stage cutting pattern with trimming. 


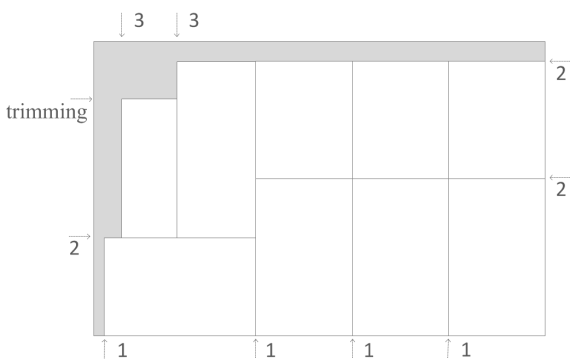

The problem has firstly been addressed by [2] and a mixed integer programming model was proposed, however no computational experiments have been conducted and a further analysis has been indicated as future work. In this study we propose to combine the model proposed in [2] with an adaptation of the heuristic proposed by [1] for the generation of cutting patterns.

\section{Integer Programming Model}

During the production process, the manufactured products must be identified by a reference in accordance to its characteristics in what concerns weaving $t \in T ; T=$ $\{1, \ldots, T\}$, dyeing $c \in C ; C=\{1, \ldots, C\}$ and printing $e \in E ; E=\{1, \ldots, E\}$. In the weaving phase, besides the minimum production quantity $\left(L_{\text {min }}^{t}\right)$ constraint, only fabric rolls with predefined widths for each weaving reference $\left(J_{t}\right)$ are produced. As previously defined, in each production phase intermediate stock can be used for each width $j$ in reference $t c e$, represented by $S_{j}^{t c e}$

In the cutting process too short cutting patterns are not desirable, thus a minimum length $P_{\text {min }}$ is defined for each cutting pattern. The cutting pattern $p$ has a length $L_{p}$, a width $W_{p}$ and is composed by $a_{i p}$ pieces of type $i$ and has a cutting process time $\left(T_{p}\right)$. In what concerns the cutting table, there is a maximum length $P_{\max }$, and a limit on the maximum height $h_{\max }^{t c e}$, defined by the maximum number of layers of fabric with reference $t c e$.

The ordered piece $i$ of reference $t c e$ is characterized by a length $l_{i}$, a width $w_{i}$ and a lower $q_{i}^{L B}$ and an upper bound $q_{i}^{U B}$ on the demand.

The following mixed integer programming model was adapted from [2] the first reference to this problem.

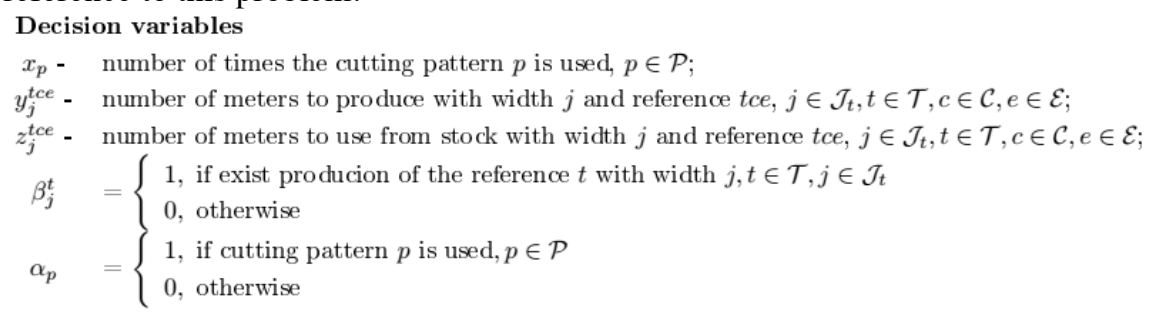




$$
\begin{aligned}
\text { Minimize } & \sum_{j t c e} K_{1 j}^{t c e} y_{j}^{t c e}+\sum_{j t c e} K_{2 j}^{t c e} z_{j}^{t c e}+\sum_{p}\left\lceil\frac{x_{p}}{h_{\text {max }}^{t c e}}\right\rceil \\
\text { Subject to: } & \sum_{c \in \mathcal{C}, e \in \mathcal{E}} y_{j}^{t c e} \geq L_{\text {min }}^{t} \cdot \beta_{j}^{t}, \quad \forall t \in \mathcal{T}, j \in \mathcal{J}_{t} \\
& \sum_{c \in \mathcal{C}, e \in \mathcal{E}} y_{j}^{t c e} \leq M \cdot \beta_{j}^{t}, \quad \forall t \in \mathcal{T}, j \in \mathcal{J}_{t} \\
& z_{j}^{t c e} \leq S_{j}^{t c e}, \quad \forall j \in \mathcal{J}_{t}, t \in \mathcal{T}, c \in \mathcal{C}, e \in \mathcal{E} ; \\
& \sum_{p: j t c e} L_{p} x_{p}-y_{j}^{t c e}-z_{j}^{t c e} \leq 0, \quad \forall j \in \mathcal{J}_{t}, t \in \mathcal{T}, c \in \mathcal{C}, e \in \mathcal{E} ; \\
& \sum_{p \in \mathcal{P}} a_{i p} x_{p} \geq q_{i}^{L B}, \quad \forall i \in \mathcal{I} ; \\
& \sum_{p \in \mathcal{P}} a_{i p} x_{p} \leq q_{i}^{U B}, \quad \forall i \in \mathcal{I} ; \\
& L_{p} x_{p}-P_{\text {min }} \alpha_{p} \geq 0, \quad \forall p \in \mathcal{P} ; \\
& x_{p}-M \alpha_{p} \leq 0, \quad \forall p \in \mathcal{P} ; \\
& x_{p} \geq 0 \text { and integer, } \quad \forall p \in \mathcal{P} ; \\
& \beta_{j}^{t} \in\{0,1\} \quad \forall j \in \mathcal{J}_{t}, t \in \mathcal{T} ; \\
& y_{j}^{t c e} \geq 0, \quad z_{j}^{t c e} \geq 0, \quad \forall j \in \mathcal{J}_{t}, t \in \mathcal{T}, c \in \mathcal{C}, e \in \mathcal{E} ; \\
& \alpha_{p} \in\{0,1\}, \quad \forall p \in \mathcal{P} .
\end{aligned}
$$

The objective function expressed by equation (1), aims at minimizing the fabric cost and the wastage. The fabric cost is composed by the weighted sum of the cost to produce the fabric throughout the production process $\left(K_{1 j}^{t c e}\right)$, the cost of using fabric from stock $\left(K_{2 j}^{t c e}\right)$ and the wastage is considered in the number of cutting tables for each reference $t c e$. The division of $\left(x_{p}\right)$ by the maximum number of layers in each cutting table $h_{\text {max }}^{\text {tce }}$ determines the total number of cutting tables for reference $t c e$, in which cutting pattern $P$ is used.

In constraints (2) and (3) it is ensured that the total production is at least of $L_{\min }^{t}$, if there exists production of the fabric roll with weaving reference $t$ and width $j$ $\left(\beta_{j}^{t}=1\right)$.

Constraint (4) ensures that the quantities used from stock for the reference tce and width $j$ effectively exist.

The overall length used in the cutting process with reference tce and width $j$ is obtained from the production in earlier phases $\left(y_{j}^{t c e}\right)$ or from stock $\left(z_{j}^{t c e}\right)$. This constraint is ensured in (5).

One of the main objectives of the model is to fulfil the orders with minimum waste and constraints (6) and (7) ensure the minimum and maximum production for each piece $i$ for the combination $t c e$.

Constraints (8) and (9) ensure that, if a cutting pattern is used, it will be applied at least to a minimum length of fabric $\left(P_{\text {min }}\right)$, the big $\mathrm{M}$ considered to be equal to the sum of all the ordered pieces times the corresponding demand.

The last four constraints (10)-(13) define the domains of the decision variables.

The model presented determines optimally the length of fabric with reference tce and width $j$ to be produced and/or to be used from stock, as well as the selection 
of the cutting patterns that generate the best solution, determining how many times the cutting patterns should be used.

\section{Solution Approach}

It is assumed, in the Mixed Integer Programming Model (1)-(13), that the complete set $P$ of cutting patterns is known. However, an explicit search of $P$ may be computationally impossible when $|P|$ is huge. In practice, one starts by considering the linear relaxation of (1)-(13) and works with a reasonable subset $P^{\prime} \subset P$ of columns, with a Restricted Master Problem (RMP). The linear relaxation of the RMP is solved via column generation, where the pricing subproblem is a twodimensional knapsack problem.

Attractive columns are added to the RMP derived from the pricing subproblem solution, where a new column represents a new cutting pattern and its corresponding length.

The pricing subproblem uses the dual values $(\Pi)$ associated with constraints (6), ensuring the minimum production of piece $i$. The geometrical part of the problem is considered in the pricing subproblem, where it is ensured that the pieces completely lie on the cutting pattern, do not overlap and the cutting patterns are of type guillotine.

The method adopted is based on the delayed column generation approach proposed by [4]. A heuristic is used to generate cutting patterns for each reference tce and width $j$ and, when the cutting patterns heuristically obtained do not improve the current value of the RMP, the pricing subproblem is solved to optimality for 2stage cutting patterns (with trimming) and the length of the cutting table is set to the pattern length.

The column generation process starts with a set of columns representative of each piece type $i$ with reference $t c e$ and width $j$. The initial cutting patterns are composed by one piece of type $i$ with reference $t c e$ and the cutting pattern length is equal to the maximum length of the cutting table $\left(P_{\max }\right)$. The initial columns/patterns often considered the maximum number of pieces that fit in the cutting pattern, however, in order to avoid unfeasible solutions due to the upper bound constraint, we consider only one-piece patterns.

The role of the pricing subproblem is to provide cutting patterns that price out profitably or to prove that none exists and a separate pricing subproblem is considered for each reference $t c e$ and width $j$. Firstly the greedy heuristic proposed by [1] is used. A priority criterion is used for piece selection: the pieces with the highest dual value and highest demand are firstly chosen. The heuristic allows the generation of cutting patterns of type two-stage with trimming and three-stage, with and without trimming and with "half-pieces".

In each iteration of the column generation process the current value of the linear relaxation of the $\operatorname{RMP}\left(Z_{R M P}^{l r}\right)$ is updated. 
When the heuristic does not improve the value of $\left(Z_{R M P}^{l r}\right)$, the pricing subproblem is solved by the exact method proposed by [3]. The pricing subproblem is now composed by a sequence of two knapsack problem types, allowing the generation of cutting patterns of the type two-stage with trimming. The first knapsack problem type creates stripes, while the second knapsack aggregates the horizontal stripes generating a cutting pattern. A total of $n+1$ knapsack problems are solved, it should be noted that this method of solving a set of knapsack problems will be applied as often as the references tce and with width $j$. The column generation process ends when the value of $\left(Z_{R M P}^{l r}\right)$ is no longer improved.

As the integrality of the variables of the initial formulation (1)-(13) was relaxed and the linear relaxation was solved via column generation, the solution obtained in the end of the column generation process has values that may not be integer. This issue is addressed by defining the decision variable $x_{p}$ as integer and $\beta_{j}^{t}$ and $\alpha_{p}$ as binaries and solving again the problem. Thus, a final integer solution is obtained. The overall solution approach is summarized in Algorithm 1.

Fig. 4. Algorithm 1.

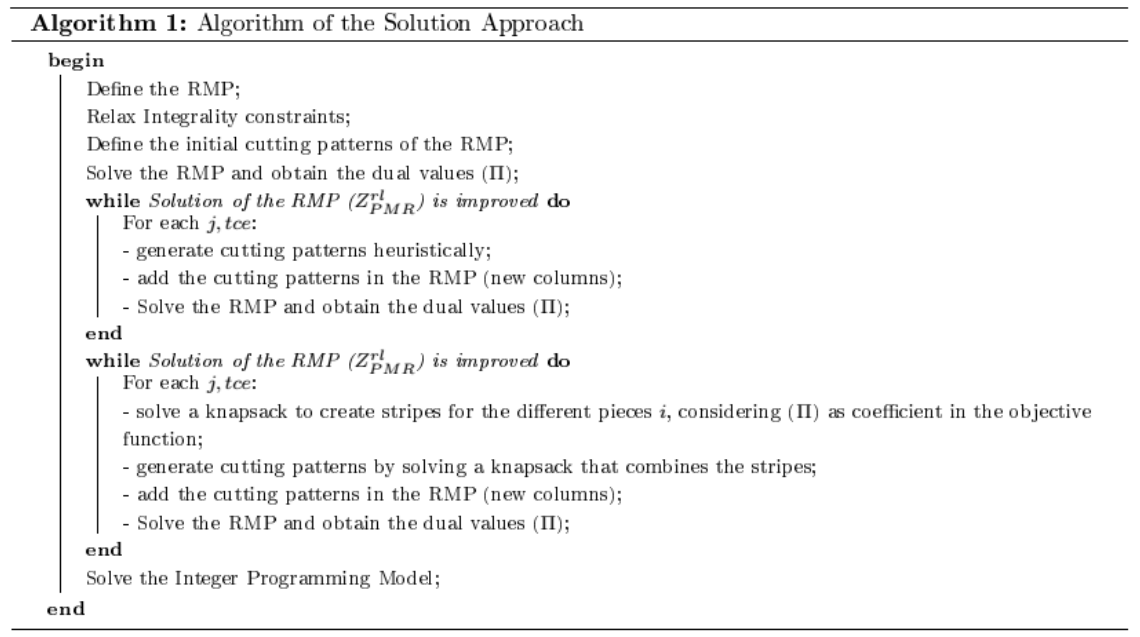

\section{Computational Results}

Computational experiments have been conducted on a real instance from the home textile manufacturing company. The demand is of 18 piece types and 4 different references tce. This demand may be cut from 20 different fabric roll types. The main objective of the computational experiments was to evaluate the sensibility of the heuristic by considering different parameters. 
The solution approach has been implemented in $\mathrm{C}++$ in Microsoft Visual Studio with the solver IBM ILOG Cplex 12.6. The computational experiments were performed in an Intel Core i7 with 4GB of RAM.

Given the uncertainty around some of the required parameters, that not even the home textile manufacturing company was able to specify, a sensitivity analysis on was run, with those parameters set for a base scenario and varied during the computational experiments.

The cost of using a fabric roll from stock $\left(K_{1 j}^{t c e}\right)$ has been defined as twice the cost of producing the fabric roll $\left(K_{2 j}^{t c e}\right)$. The maximum number of layers of fabric on the cutting table $\left(h_{\max }^{\text {tce }}\right)$ has been set to 20 . The fabric rolls in stock have been fixed to zero for all references tce and widths $j$. The minimum length of fabric roll to produce in the weaving phase has been defined as $10 \%$ of the total length of the pieces. The minimum length at which a cutting pattern can be applied $\left(P_{\min }\right)$ has been defined as zero and the maximum length of the cutting table $\left(P_{\max }\right)$ is set to $1500 \mathrm{~cm}$, which corresponds to its real length.

The parameters $h_{\max }^{t c e}$ and $P_{\max }$ are kept constant in the different scenarios. In Table 1 the 6 tested scenarios are presented.

Table 1. Parameters of the different scenarios.

\begin{tabular}{cccccccc}
\hline Scenario & $\boldsymbol{K}_{\mathbf{1}}^{\text {tce }}$ & $\boldsymbol{K}_{\boldsymbol{2} \boldsymbol{j}}^{\text {tce }}$ & $\boldsymbol{h}_{\boldsymbol{m a x}}^{\text {tce }}$ & $\boldsymbol{S}_{\boldsymbol{j}}^{\text {tce }}$ & $\boldsymbol{L}_{\min }^{\boldsymbol{t}}$ & $\boldsymbol{P}_{\min }$ & $\boldsymbol{P}_{\max }$ \\
\hline Base & 1 & 2 & 20 & 0 & 36532 & 0 & 1500 \\
$\mathbf{1}$ & 1 & 2 & 20 & 0 & 36532 & 6000 & 1500 \\
$\mathbf{2}$ & 1 & 2 & 20 & 0 & 0 & 0 & 1500 \\
$\mathbf{3}$ & 1 & 2 & 20 & 0 & 730655 & 0 & 1500 \\
$\mathbf{4}$ & 1 & 1 & 20 & 913 & 36532 & 0 & 1500 \\
$\mathbf{5}$ & 2 & 1 & 20 & 913 & 36532 & 0 & 1500 \\
\hline
\end{tabular}

The computational results are summarized in Table 2. The solutions obtained during the column generation process using only the heuristic to solve the subproblem are presented in column $S P_{\text {heur }}$. Column $S P_{\text {heur- }} I P$ presents the solutions obtained for the linear relaxation of the RMP after the exact method has been used for solving the subproblem. The number of columns generated by the subproblems for each method is represented by $n_{c o l}$, the integer solution is represented by $Z_{R M P}^{m i p}$, gap $_{\text {mip }}$ relates the value of RMP relaxed with the integer solution, $\Sigma Y$ is the total length to be produced in centimeters, $\sum Z$ is the total length in centimeters of fabric to be used from stock, $N_{p}$ represents the number of different cutting patterns used in the solution and $\mathrm{Avg}_{n p}$ is the average number of times the cutting pattern is used.

The first analysis comprises the $P_{\min }$ parameter, that is equal to zero in the base scenario and in scenario 1 is equal to 6000 (four times the length of the cutting table). An increase of the minimum length that a cutting pattern can be applied means an increase of the frequency of utilization of the cutting pattern, thus reduc- 
ing the setup time, but increasing the waste. This conclusion is corroborated by the value of the objective function that increased with the increase of $P_{\text {min }}$.

In scenarios 2 and $3 L_{\mathrm{min}}^{t}$ was fixed to zero and to $20 \%$ of the total length of the pieces, respectively. The increase of the minimum length of production in the weaving phase also increases the waste, since the geometric combinations are reduced. It is also interesting to notice that an increase on the value of $L_{\min }^{t}$ reduces the number of different widths that are used for each reference tce.

In scenarios 4 and 5 the fabric roll stock was set to 913 for each type of fabric roll. This value was obtained by weighing $5 \%$ of the needs of each reference $t$. In this new instance the model can choose between production and stock. The coefficients of the objective function were also changed in order to analyze the behavior of the model. As expected the solution obtained uses more fabric rolls from stock when the costs are smaller.

Table 2. Results considering different scenarios on the parameters.

\begin{tabular}{|c|c|c|c|c|c|c|c|c|c|c|c|}
\hline \multirow[t]{2}{*}{ Scenario } & \multicolumn{2}{|c|}{$S P_{\text {heur }}$} & \multicolumn{2}{|c|}{$S P_{\text {heur_I }} I P$} & \multicolumn{7}{|c|}{ Final Solution } \\
\hline & $Z_{R M P}^{l r}$ & $n_{c o l}$ & $Z_{R M P}^{l r}$ & $n_{c o l}$ & $Z_{R M P}^{m i p}$ & $g a p_{\text {mip }}$ & $\sum Y$ & $\sum Z$ & $N_{p}$ & & Total \\
\hline Base & 518031 & 200 & 517396 & 120 & 521029 & 0.7 & 521012 & 0 & 24 & 14.5 & 64 \\
\hline 1 & 518032 & 200 & 517396 & 120 & 521347 & 0.8 & 521330 & 0 & 17 & 20.5 & 15 \\
\hline 2 & 518063 & 200 & 517462 & 80 & 520915 & 0.7 & 520898 & 0 & 28 & 12.5 & 71 \\
\hline 3 & 518063 & 200 & 517396 & 120 & 540336 & 4.3 & 540319 & 0 & 20 & 17.5 & 64 \\
\hline 4 & 518063 & 200 & 517396 & 120 & 521029 & 0.7 & 517360 & 3652 & 24 & 14.5 & 64 \\
\hline 5 & 1024159 & 200 & 1023123 & 120 & 1037476 & 1.4 & 516447 & 4564 & 23 & 15.2 & 65 \\
\hline
\end{tabular}

\section{Conclusions}

This paper addresses the optimization of the production planning problem integrated with the cutting problem in a home textile manufacturing company. The complete production process was analyzed and a solution approach is proposed based on the column generation technique.

The methodology developed is able to decide the quantity of fabric that should be produced in each production phase, from each reference and the quantity of fabric that should be used from stock and the respective cutting patterns that should be applied to obtain the pieces and the corresponding quantities.

Preliminary computational experiments have been conducted on a real instance and an analysis of the sensibility of the proposed approach to different parameters tuning was performed, one can conclude that the proposed heuristic is highly dependent on the different costs considered in the objective function. 
The future research will focus on the improvement of the column generation procedure adopted and on the development of a new strategy to obtain integer solutions.

\section{Acknowledgements}

The first author is grateful to FCT - Fundação para a Ciência e Tecnologia (Portuguese Foundation for Science and Technology) - for awarding the grant SFRH / BPD/98981/2013.

\section{References}

[1] Almeida, RN., 2014. Redução dos desperdícios no processo de corte em empresas Têxtil-Lar. Master thesis, FEUP.

[2] Cerqueira, B., 2013. Kaizen na indústria têxtil - Uma abordagem ao aumento de produtividade e redução de desperdício.

[3] Gilmore PC. \& Gomory RE., 1965. Multistage cutting stock problems of two and more dimensions. Operations Research, 13(1), 94-120.

[4] Oliveira, JF. \& Ferreira, JS:, 1994. A faster variant of the Gilmore and Gomory technique for cutting stock problems. Jorbel, 34(1), 23-38. 